\title{
The mesothelioma enigma
}

\author{
Raja M. Flores, MD
}

See related article on pages 1539-47.

Many experts declare that refined surgical technique, improved chemotherapy, and advances in radiotherapy have greatly helped advance the field of mesothelioma over the decades. Unfortunately, this is not reflected in median survivals. Over the past 75 years, very little has changed with regard to long-term outcome in mesothelioma. The median survivals of 21 months for epithelioid and 12 months for mixed and sarcomatoid histology have been reported for patients from as far back as 1939 and still look lamentably similar to results from today. ${ }^{1}$ Modern studies tend to focus on patients who have passed the rigorous treatment selection process while overlooking the majority who have not.

The study by Frischknecht and colleagues ${ }^{2}$ assessed the prognostic ability of excision repair cross-complementary group 1 (ERCC1) and ribonucleotide reductase subunit M1 (RRM1) measured by pretreatment tissue biopsy in patients who underwent induction chemotherapy followed by extrapleural pneumonectomy (EPP). The bottom line of the study was that high ERCC1 expression was bad and high RRM1 was good in this highly select group of patients with regard to prognostication. These findings were statistically significant by univariate analyses.

The study suffers from several weak points. The multivariate model included only T stage (not T2 vs T3), ERCC1, and RRM1. A multivariate mesothelioma model without histology as a variable raises validity questions because histology is universally regarded as the single greatest influence on outcome. In addition, the authors did not include age, gender, and nodal status in the multivariate model, which were statistically significant in the univariate analyses. These confounding variables could alter the observed statistical significance of ERCC1 and RRM1 in the final multivariate model. The primary end point was freedom from recurrence. This end point is suboptimal

From the Department of Thoracic Surgery, Mount Sinai Health System, Icahn School of Medicine at Mount Sinai, New York, NY.

Disclosures: Author has nothing to disclose with regard to commercial support.

Received for publication Feb 20, 2015; accepted for publication Feb 20, 2015; available ahead of print March 24, 2015.

Address for reprints: Raja M. Flores, MD, Department of Thoracic Surgery, Mount Sinai Health System, Icahn School of Medicine at Mount Sinai, One Gustave L. Levy Place, Box 1023, New York, NY 10029 (E-mail: raja.flores@mountsinai. org).

J Thorac Cardiovasc Surg 2015;149:1548-9

0022-5223/\$36.00

Copyright (c) 2015 by The American Association for Thoracic Surgery http://dx.doi.org/10.1016/j.jtcvs.2015.02.036 and fraught with inaccuracies, which is why most end points in mesothelioma studies focus on overall survival. Radiologic and pathologic staging for mesothelioma is unreliable because of the diffuse nature of the tumor; therefore, homogeneously staged patient groups are difficult to accurately identify. This also relates to radiologic postoperative recurrence,

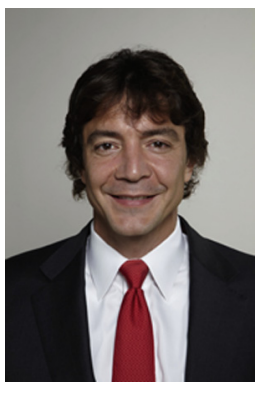
which can be confused with postsurgical change, talc reaction, and radiation fibrosis $(\sim 60 \%$ had radiotherapy). Of note, radiotherapy (a local recurrence treatment) was not significant in this study in which freedom from recurrence was the end point. Response Evaluation Criteria In Solid Tumors criteria are subject to radiologist bias and cannot overcome radiologic shortcomings intrinsic to this disease. However, these deficiencies are not unique to this study but are common to all mesothelioma studies.

The predictive ability of ERCC1 and RRM1 would be useful if they could help us administer chemotherapy when it is effective and avoid chemotherapy when it is futile, as exists in lung cancer, but predictability was not demonstrated. The prognostic role of ERCC1 and RRM1, although statistically significant in this study, is not clinically relevant at this time.

Mesothelioma studies have devolved into reports on subsets of subsets of subsets of patients. The denominator in the study by Frischknecht and colleagues ${ }^{2}$ includes patients who did not tolerate induction chemotherapy, patients who were unresectable at the time of surgery, and operative deaths, just to name a few variables that affect overall survival. This study focuses on the patients who survived the natural selection process, thereby ignoring the intentto-treat principle. This of course selects for patients who will have a better outcome but not necessarily as a result of treatment. The omission of patients who failed the rigorous initial treatment regimen probably has more of an impact on prognostic outcome than ERCC1 or RRM1. Again, these shortcomings are not just limited to this study but the majority of multimodality mesothelioma studies.

This study mistakenly gives the impression that induction chemotherapy followed by EPP is the standard treatment for mesothelioma. Many studies have investigated induction versus adjuvant chemotherapy, surgical treatment by EPP, pleurectomy decortication or talc pleurodesis, radiotherapy before and after surgery, and intracavitary therapy, and yet there is still no consensus. Surgical treatment varies greatly and is fiercely debated even after a valiant 
but failed randomized trial comparing surgery by EPP with no surgery. Although debate rages over whether surgery is effective, it would make sense to favor the procedure with the lowest morbidity and mortality. Median survivals after EPP and pleurectomy decortication are similar, with outlying long-term survivors observed in both groups. However, the operative mortality appears 3 -fold higher with EPP than with pleurectomy decortication. ${ }^{3}$ This difference is even more pronounced at the 90-day mark, mounting more evidence in favor of reserving EPP as a procedure of last resort or completely eliminating it. Could a shift in surgical procedure have a larger impact on outcome greater than any other investigative treatment or biomarker currently available? Although the role of ERCC1 and RRM1 are interesting, our efforts also should focus on issues that would have a greater immediate impact on outcome. Would the patients in this study have fared better with pleurectomy decortication or with no surgery at all? The primary end point of this study looks at the role of prognostic biomarkers, but the bigger surgical picture is lost. I still perform EPP in select cases, but my faith is fading. Mesothelioma is an enigma, but the surgical treatment should not be.

\section{References}

1. McCormack PM, Nagasaki F, Hilaris BS, Martini N. Surgical treatment of pleural mesothelioma. J Thorac Cardiovasc Surg. 1982;84:834-42.

2. Frischknecht L, Meerang M, Soltermann A, Stahel R, Moch H, Seifert B, et al. Importance of ERCC1 and RRM1 as prognostic biomarkers in malignant pleural mesothelioma treated with platinum based induction chemotherapy followed by surgery. J Thorac Cardiovasc Surg. 2015;149: 1539-47.

3. Taioli E, Wolf AS, Flores RM. Meta-analysis of survival after pleurectomy decortication versus extrapleural pneumonectomy in mesothelioma. Ann Thorac Surg. 2015;99:472-80. 